Journal for ImmunoTherapy of Cancer

\title{
DNA methylation-based signature of CD8+ tumor-infiltrating lymphocytes enables evaluation of immune response and prognosis in colorectal cancer
}

Qi Zou, ${ }^{1,2,3}$ Xiaolin Wang, ${ }^{1}$ Donglin Ren, ${ }^{1,3}$ Bang Hu, ${ }^{1,3}$ Guannan Tang, ${ }^{1}$ Yu Zhang, ${ }^{1}$ Meijin Huang, ${ }^{1,2}$ Rish K Pai, ${ }^{4}$ Daniel D Buchanan, ${ }^{5,6,7}$ Aung Ko Win, ${ }^{8}$ Polly A Newcomb, ${ }^{9,10}$ William M Grady, ${ }^{11,12}$ Huichuan Yu (D) , ${ }^{1}$ Yanxin Luo (D) ${ }^{1,2}$

To cite: Zou $Q$, Wang $X$, Ren D, et al. DNA methylationbased signature of CD8+ tumor-infiltrating lymphocytes enables evaluation of immune response and prognosis in colorectal cancer. Journal for ImmunoTherapy of Cancer 2021;9:e002671. doi:10.1136/ jitc-2021-002671

- Additional supplemental material is published online only. To view, please visit the journal online (http://dx.doi.org/10. 1136/jitc-2021-002671).

QZ, XW and DR contributed equally.

$\mathrm{HY}$ and $\mathrm{YL}$ contributed equally.

$\mathrm{HY}$ and $\mathrm{YL}$ are joint senior authors.

Accepted 11 August 2021

\section{Check for updates}

(c) Author(s) (or their employer(s)) 2021. Re-use permitted under CC BY-NC. No commercial re-use. See rights and permissions. Published by BMJ.

For numbered affiliations see end of article.

\section{Correspondence to} Dr Huichuan Yu; yuhch5@mail.sysu.edu.cn

Dr Yanxin Luo; luoyx25@mail.sysu.edu.cn

\section{ABSTRACT}

Background Tumor-infiltrating lymphocytes (TILs), especially CD8 + TILs, can be used for predicting immunotherapy responsiveness and survival outcome. However, the evaluation of CD8 + TILs currently relies on histopathological methodology with high variability. We therefore aimed to develop a DNA methylation signature for CD8 + TILs (CD8 + MeTIL) that could evaluate immune response and prognosis in colorectal cancer (CRC). Methods A CD8 + MeTIL signature score was constructed by using CD8 + T cell-specific differentially methylated positions (DMPs) that were identified from Illumina EPIC methylation arrays. Immune cells, colon epithelial cells, and two CRC cohorts ( $\mathrm{n}=282$ and 335) were used to develop a PCR-based assay for quantitative analysis of DNA methylation at single-base resolution (QASM) to determine CD8 + MeTIL signature score .

Results Three CD8 + T cell-specific DMPs were identified to construct the CD8 + MeTIL signature score, which showed a dramatic discriminability between CD8 + T cells and other cells. The QASM assay we developed for CD8 + MeTIL markers could measure CD8 + TILs distributions in a fully quantitative, accurate, and simple manner. The CD8 + MeTIL score determined by QASM assay showed a strong association with histopathology-based CD8 + TIL counts and a gene expression-based immune marker. Furthermore, the low CD8 + MeTIL score (enriched CD8 + TILs) was associated with MSI-H tumors and predicted better survival in CRC cohorts.

Conclusions This study developed a quantitative DNA methylation-based signature that was reliable to evaluate CD8 + TILs and prognosis in CRC. This approach has the potential to be a tool for investigations on CD8 + TILS and a biomarker for therapeutic approaches, including immunotherapy.

\section{INTRODUCTION}

Colorectal cancer (CRC) is one of the leading causes of cancer-related death worldwide. ${ }^{12}$ Immunotherapy is playing an increasingly important role in treating CRC in both resectable and non-resectable patients. ${ }^{3}$ Immune checkpoint inhibitors can boost $\mathrm{T}$ cells to attack tumor cells and prolong patient survival. $^{4-6}$ Meanwhile, immunotherapy for CRC remains challenging because of its heterogeneous immune response. ${ }^{78}$ Tumorinfiltrating lymphocytes (TILs) are associated with tumor immune response and can be applied to predict the response to immunotherapy and survival outcome. ${ }^{9-11}$ Among them, CD8 + TILs kill cancer cells in a cytotoxic manner and are recognized to affect prognosis, immunotherapy response, and survival outcome in CRC. ${ }^{12-14}$

Multiple morphology-based methods have been established and widely validated to evaluate TILs in a reproducible way, including the recommendations by an international TILs working group ${ }^{15}$ and an immunoscore assay. ${ }^{16}$ In addition, some gene expressionbased immune markers were developed to be an adjunct method to assess CD8+ TILs, ${ }^{17-19}$ including the $C D 8 B$ gene expression marker panel. Moreover, some DNA methylationbased markers for TIL (MeTIL) were recently identified from epigenome-wide analyses in breast cancer, showing good performance in the evaluation of the local tumor immune response and potential to improve the prognostic accuracy in patients with multiple cancer. $^{11}$

DNA methylation is tissue-specific and plays a decisive role in tissue differentiation. ${ }^{20} 21$ Thus, DNA methylation profiling varies in cells from different tissues and organs, and it has the potential to be a marker to recognize different cells. ${ }^{22}$ Among them, the methylation of CpG shores within intermediate-CpG density regions is highly conserved and can discriminate tissue types regardless of species of origin. ${ }^{23}$ In addition, dramatic hypomethylation of partially methylated domains (PMDs) occurs during lymphocyte activation 
and proliferation, which can be predicted by solo-WCGWs (CpGs flanked by A or T in PMD context) that are mostly located in low-CpG density regions. ${ }^{24}$ Unfortunately, these areas of DNA methylation that have the potential to distinguish immune cells from other cells are characterized by low CpG density, ${ }^{25}$ which technically hinders the quantitative PCR (qPCR)-based assays in determining the methylation in these regions. To achieve a robust singlebase specificity using PCR, we developed a novel assay to best use these low CpG-density methylations for developing biomarkers. ${ }^{26}$

In this study, we aimed to analyze the genome-wide DNA methylation profile of immune cells and colon epithelial cells to identify CD8 + T cell-specific differentially methylated positions (DMPs). Then, we constructed a CD8 + MeTIL signature score by using these DMPs to assess CD8 + TILs and survival outcomes in CRC and other cancers. Finally, we developed a qPCR-based assay to determine this score by using low amounts of DNA from formalin-fixed, paraffin-embedded (FFPE) tumor tissue. We demonstrated the utility of this methodology in CD8 + TIL evaluation.

\section{MATERIALS AND METHODS \\ Study design}

This study aimed to identify DNA methylation-based biomarkers that can assess CD8 + TILs in CRC. First, we identified CD8 + T cell-specific DMPs from the discovery set composed of CD8 + T cells, other immune cells, and cancerous and normal colorectal epithelial cells with EPIC methylation array datasets. We then selected DMPs and constructed a CD8 + MeTIL signature score model with a machine learning algorithm. We further developed a QASM assay to test selected DMPs in a qPCRbased manner at single-base resolution. Finally, we tested the selected DMPs in multiple cohorts with this method, compared them with $C D 8 B$ gene expression assay and immunohistochemical (IHC) assay for CD8 + TILs counts, and investigated their prognostic value in CRC and other cancers.

\section{Patient cohorts and tumor samples}

Two cohorts with CRC tumor samples from Colon Cancer Family Registry (CCFR) study and Sixth Affiliated Hospital of Sun Yat-sen University (SAH-SYSU) tumor bank were applied in this study. A description of the populations in CCFR study ${ }^{27}$ and SAH-SYSU samples ${ }^{29}{ }^{30}$ has been published previously. Briefly, the CCFR study cohort included 335 patients with pathologically confirmed CRC at Seattle, Ontario, and Mayo CCFR sites, and the SAHSYSU cohort consisted of 282 patients with pathologically confirmed CRC at SAH-SYSU from 2008 to 2012. All cases underwent radical-intent resection of the primary tumor. Patients with any prior anticancer treatment, concurrent cancer other than CRC, chronic inflammatory bowel disease, familial adenomatous polyposis, and whose DNA sample was degraded or not available were excluded. See online supplemental tables for the baseline characteristics of these cohorts. All samples used in this study were reviewed by pathologists to confirm the diagnosis and ensure that the tumor samples had more than $60 \%$ tumor content. To externally validate the findings in other cancer types, we used 24 The Cancer Genome Atlas (TCGA) cohorts with InfiniumHuman Methylation 450K BeadChip (450K methylation array) profile. The workflow of cohort disposition is summarized in figure 1 .

\section{Colon epithelial cells and immune cells}

All cancerous and normal colon cell lines (RKO, SW48, HCT8, HCT15, and NCM460) were obtained from American Type Culture Collection. Human-derived immune cells, including CD4+, CD8+, monocytes, and natural killer (NK) and B cells (1505, 1508-1510, 1513; LDE Biotechnology Co., Guangzhou) were enriched by magnetic-activated cell sorting.

\section{Screen of CD8+ T cell-specific DMPs in CRC}

A discovery set $(n=167)$ composed of CD8 $+T$ cells, nonCD8 + immune cells, cancerous and normal colorectal epithelial cells with Illumina Infinium MethylationEPIC Beadchip (EPIC methylation array) methylation profile data was obtained from Gene Expression Omnibus datasets with identifiers: GSE103541, GSE118144, GSE118972, GSE111681, and GSE122126. ${ }^{31-34}$ The characteristics of these samples are summarized in the online supplemental tables. The 'minfi' R package and Combat tool were used to perform raw data processing, batch effect correction, and DMP analysis as previously described.$^{30}{ }^{35} 36$ To identify CD8 + T cell-specific DMPs that can distinguish CD8 + $\mathrm{T}$ cells from non-CD $8+$ immune cells and cancerous and normal colorectal epithelial cells, we performed DMP analysis using their methylation data and selected the topranked candidate.

\section{Nucleic acid isolation and bisulfite conversion}

Genomic DNA was extracted from $20 \mathrm{mg}$ fresh-frozen tissues using QIAamp DNA Mini Kit (Qiagen, 51306) and treated with sodium bisulfite using EZ DNA Methylation Kit (Zymo, D5002). RNA was extracted from $20 \mathrm{mg}$ fresh-frozen tissues using Rneasy Plus Mini Kit (QIAGEN, 74134).

\section{EPIC methylation array and bisulfite pyrosequencing}

Evaluation of genome-wide DNA methylation was performed in 45 fresh-frozen tumor samples from the SAH-SYSU cohort using EPIC methylation array following the manufacturer's protocol. The raw data were processed with the method described in the screen of CD8 + T cellspecific DMPs. We also evaluated the methylation of selected CpGs in this cohort by bisulfite pyrosequencing as described in the online supplemental materials.

qPCR-based quantitative analysis for single-base methylation (QASM)

The methylation percentage of each candidate $\mathrm{CpG}$ site was determined in multiple cohorts using a 




Figure 1 Flow diagram of the model generation and cohort disposition in this study. A discovery set with EPIC methylation array data of CD8 + T cells, cancerous and normal colorectal epithelial cells and other human-derived immune cells was used to construct a CD8 + MeTIL signature. The SAH-SYSU and CCFR study cohorts were two in-house cohorts with patients with pathologically confirmed colorectal cancer. The SAH-SYSU cohort consists of 282 cases that were used to develop a PCRbased assay for quantitative analysis of DNA methylation at single-base resolution (QASM) to determine CD8 + MeTIL signature score, validate its role in the evaluation of CD8 + TILs-based immune response, and test its prognostic value e. The CCFR cohort consists of 335 cases from CCFR study, in which the distribution of CD8 + MeTIL score by molecular phenotypes was analyzed and its prognostic value was further validated. The TCGA cohorts with 24 cancers whose methylation array data were available for 450K-specific CD8 + MeTIL score were used to explore the value of CD8 + MeTIL signature in other cancers. CCFR, Colon Cancer Family Registry; DMP, differentially methylated position. SAH-SYSU, Sixth Affiliated Hospital of Sun Yatsen University.

MethyLight ${ }^{37}$-based QASM assay that has been developed and validated in our previous work. ${ }^{26}$ In short, the bisulfiteconverted DNA was amplified, in which we exploited the locus-specific PCR primers flanking a pair of methylated and unmethylated probes labeled with the fluorescent dyes 6-carboxyfluorescein (6-FAM) and 2-chloro-7phen yl-1,4-dichloro-6-carboxyfluorescein (VIC), respectively. The methylation percentage was calculated by methylation/(methylation +unmethylation) $\times 100 \%$. QASM was performed using the Applied Biosystems QuantStudio 7 Flex Real-Time PCR System (Thermo). The detailed procedure of QASM assay for CD8 + MeTIL markers can be found in the online supplemental materials.

\section{IHC assessment of CD8+ TILs (CD8+ PaTIL)}

The FFPE tumor blocks were cut into $4 \mu \mathrm{m}$ sections. The deparaffinized slides were incubated with monoclonal anti-CD8 antibody (clone C8/144B; DAKO, Kyoto, Japan, SK201) at 1:100 dilution for 16 hours at $4^{\circ} \mathrm{C}$. Subsequently, the secondary antibody conjugated with horseradish peroxidase (Envision + Dual Link Kit, DAKO, K5007) was applied. IHC assessment of CD8 + TILs (CD8 + PaTIL) 
was performed following the method described by Fortis et $a .^{38}$ Briefly, visual enumeration in five representative fields $\left(0.1255 \mathrm{~mm}^{2}\right.$ per field) was performed by two independent pathologists, and the mean counts of CD8 + TILs were recorded.

\section{CD8B gene expression assay for CD8+ TILs (CD8+ ExTIL)}

Total RNA extracted from fresh-frozen tumors was reversely transcribed by ReverTra Ace qPCR RT Master Mix (TOYOBO, FSQ-201) according to the manufacturer's protocol. Then, CD8B mRNA expression was quantified by qPCR assay. The primer sequence can be found in the online supplemental tables.

\section{Molecular phenotyping}

Tumor characteristics of microsatellite status, somatic mutations in KRAS codons 12 and 13, BRAF p.V600E mutation, and $\mathrm{CpG}$ island methylator phenotype (CIMP) in each cohort were determined as previously described. ${ }^{27} 39-41$

\section{Statistical analysis}

Differences between groups were evaluated using Student's t-test, Mann-Whitney test or $\chi^{2}$ test. Consistencies between results were analyzed using Pearson's correlation analysis. The maximally selected rank statistics were applied to determine the optimal cut-off of variables in the survival analysis. Cox regression analysis and Kaplan-Meier survival curves with log-rank tests were used to compare overall survival (OS). Gene Ontology (GO) enrichment analysis was performed using 'GOfuncR' package. All the statistical analyses were conducted with R V.3.6.1. All $p$ values were calculated with two-tailed tests and considered significant when $\mathrm{p}<0.05$.

\section{RESULTS}

\section{DNA methylation signature of CD8+ TILs in colorectal tumors}

The outline of the study is shown in figure 1. To develop the CD8 + MeTIL signature, we first compared the methylation signature of $\mathrm{CD} 8+\mathrm{T}$ cells with those of cancerous and normal colorectal epithelial cells and other humanderived immune cells, including CD4 + T cells, B cells, granulocytes, and monocytes, using their genomic methylation data profiled by EPIC array (see online supplemental materials for details). ${ }^{31-34}$ We identified 73 CD $8+$ $\mathrm{T}$ cell-specific DMPs that were highly differentially methylated between CD8 $+\mathrm{T}$ cells and other cells in this discovery step (figure 2A and online supplemental material 1).

To confirm the results in the discovery step, we analyzed the biological features of these CD8 $+\mathrm{T}$ cell-specific DMPs. Most CpGs were located in the intragenic region (80.6\%, 59 probes), including CD8A, CD8B, CD96, and other functional genes in $\mathrm{CD} 8+\mathrm{T}$ cell (online supplemental material 1), and a few ones were intergenic CpGs (19.2\%, 14 probes). We further performed $\mathrm{GO}$ analysis for intragenic GpGs and found multiple immune-assoicated
GO terms were top-ranked (figure 2C). This finding confirmed the strong association of identified CpGs with CD8 + T cells.

Next, we analyzed the genomic features of these 73 CpGs. The majority were located in low-CpG density $(60.3 \%, 44$ probes) and intermediate-CpG density $(34.2 \%$, 25 probes) regions, and few CpGs (5.5\%, 4 probes) were located in the high-CpG density region (CpG island) (figure 2B). Of note, 6 CpGs were solo-WCGW CpGs, and 10 CpGs were located in common PMD, according to the previous genomic annotations. ${ }^{24}$

To achieve a shrinking panel that can be easily accessible, we applied the least absolute shrinkage and selection operator (LASSO) machine learning approach to select CpGs that can best represent our list of 73 CD8 + $\mathrm{T}$ cell-specific CpGs (online supplemental material 1 ). The final signature, named CD8 + MeTIL score, included three CpGs (cg02430840, cg06113913, and cg12673499) located in the low-CpG density region. We constructed a CD8 + MeTIL score with their $\beta$ values and coefficients in LASSO regression: $\operatorname{cg} 02430840 \times 0.28724+\operatorname{cg} 061139$ $13 \times 0.40171+\operatorname{cg} 12673499 \times 0.45016$. This score can theoretically adapt values between 0.00 and 1.14 , while we received CD8 + MeTIL scores between 0.71 and 1.00 in the discovery cohort.

To explore the distinguishability of the CD8 + MeTIL score, we grouped cells by unsupervised hierarchical cluster analysis using the three CpGs. We found that CD8 + T cells could be well distinguished from other cell types by assessing these three CpGs (figure 2D). Of note, the CD8 + MeTIL score was dramatically lower in CD8 + T cells than that in any of cancerous and normal colorectal epithelial cells, CD4 + T cells, B cells, granulocytes and monocytes (all $\Delta \beta>0.4$, all $\beta$ fold change $>3.0$, all $p<0.001$; figure 2E). This high specificity of CD8 + MeTIL score in CD8 $+\mathrm{T}$ cells was the basis of its ability to determine the abundance of CD8 + T cells in a tumor block. Furthermore, we validated the specificity of CD8 + MeTIL score in an independent sample set with DNA methylation profile of immune cells, cancerous and normal colon epithelial cells, and other cell types that were recognized in the tumor microenvironment (online supplemental material 1).

We next sought to investigate the association between the methylation profiles of the three selected CpGs in the CD8 + MeTIL score and clinicopathological features in the SAH-SYSU cohort of 45 CRC cases with EPIC array data (figure 2F). We found that a lower CD8 + MeTIL score with less methylation in the three CpGs was associated with CRCs with low-frequency microsatellite instability (MSI-H), right-sided location, and high differentiation. These results were consistent with the findings from previous IHC-based CD8 + TIL association studies. The methylation levels of these three CpGs determined by EPIC array were significantly correlated with their methylation levels determined by bisulfite pyrosequencing (figure 3D; $r=0.7357-0.9025$, all $\mathrm{p}<0.01)$. 
A

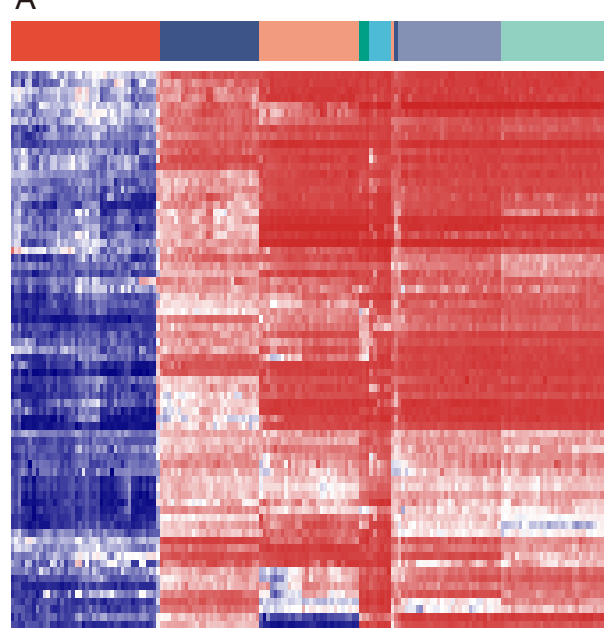

C



F

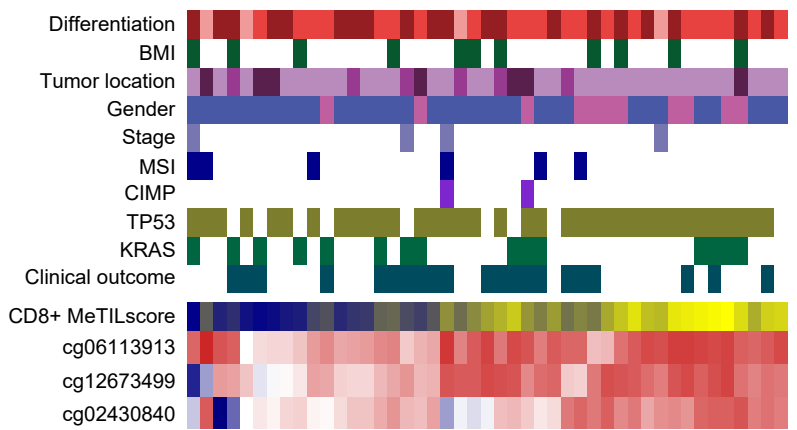

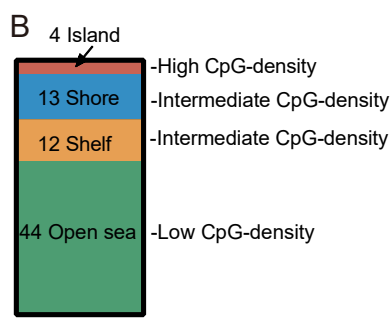



D



E




A
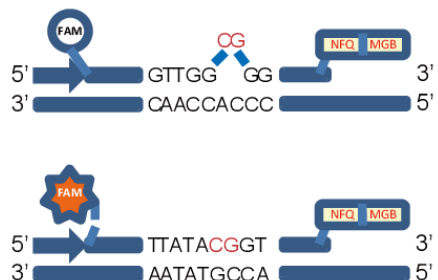

$\operatorname{cg} 06113913$

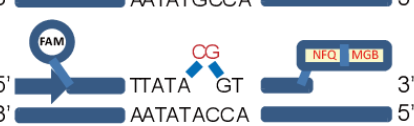

Unmethylated alleles



Figure 3 Quantitative PCR-based QASM assay for determining CD8 + MeTIL score at single-base resolution. (A) The design of QASM assay for methylation detection of each isolated CpG. Each methylation level was determined merely at probe level, in which a FAM-labeled MGB probe specifically binds to methylated allele (CpG) sequence, and the VIC-labeled probe binds to the unmethylated allele (TpG) sequence. Both alleles were amplified with one pair of primers in the same PCR reaction. (B-D) Scatter plots showing the correlations between any two of the QASM assay $(n=45)$, EPIC methylation array $(n=45)$ and bisulfite pyrosequencing $(n=12)$ in determining methylation percentage of each $\mathrm{CpG}$ targeted by cg02430840, cg06113913, and cg12673499, respectively. Pearson's test was applied and correlation coefficient $(r)$ and $p$ value for each procedure are shown. MGB, minor groove binder. 


\section{QPCR-based assay for CD8+ MeTIL score at single-base resolution}

To easily obtain CD8 + MeTIL score generated from arraybased analysis, we developed a qPCR-based assay (QASM) to determine the methylation of three selected CpGs that were located in the low-CpG density region at single-base resolution. As figure 3A displayed, the methylated and unmethylated alleles of each $\mathrm{CpG}$ were quantified at the probe level, in which methylation percentages can be determined by the signal ratio of two probes.

To validate this qPCR-based assay for determining CD8 + MeTIL score, we next compared the results of QASM assay with those of the EPIC array and pyrosequencing in the SAH-SYSU cohort. The qPCR-based and EPIC array-based methylation percentages were linearly related well in each CpG (figure 3B; $n=45, r=0.5225-0.7274$, all $\mathrm{p}<0.001$ ). In addition, the methylation percentages determined by QASM assay linearly correlated with those determined by pyrosequencing (figure $3 \mathrm{C} ; \mathrm{n}=12, \mathrm{r}=0.7170-0.9411$, all $\mathrm{p}<0.01)$. The CD8 + MeTIL scores generated from QASM assay linearly correlated with the scores generated from both the EPIC array and pyrosequencing (figure 3; $\mathrm{n}=45$ and 12 , respectively; $r=0.7357$ and 0.9025 , respectively; all $\mathrm{p}<0.001)$. Thus, QASM assay is a technically reliable tool to determine CD8 + MeTIL score.

To measure CD8 + TIL distribution with CD8 + MeTIL score, we first used QASM to determine the methylation percentages of three CpGs in different tissues and cells, including CRC tissue $(\mathrm{n}=45)$ and adjacent normal tissue $(\mathrm{n}=44)$, normal (NCM460) and cancerous (RKO, SW48, HCT8, and HCT15) colon epithelial cell lines and multiple immune cells (CD4 + T cells, monocytes, and B cells), which represented the major components of CRC tissues. The CD8 + T cells displayed a significantly lower CD8 + MeTIL score compared with other cells using the QASM assay (all score fold change $>3.0$, all $\Delta$ score $>2.3$, all $\mathrm{p}<0.001$; figure $4 \mathrm{~A}$ ) and were consistent with the results determined from the EPIC array dataset, further supporting the qPCR-based CD8 + MeTIL score as an accurate surrogate for the array-based score to determine the abundance of CD8 + T cells in tumor samples.

\section{Evaluation of CD8+ TIL distribution in CRC with CD8+ MeTIL score}

We applied this qPCR-based CD8 + MeTIL score to assess CD8 + TILs in tumor tissues and compared it with CD8 + TILs determined by IHC staining (CD8 + PaTILs) in SAHSYSU cohorts (figure 4B,C). The CD8 + PaTILs displayed a significant negative correlation with the CD8 + MeTIL score $(\mathrm{r}=-0.6385, \mathrm{p}=0.011)$. Notably, CD8 + MeTIL low group had significantly more CD8 + TIL counts in IHC staining than the CD8 + MeTIL high group $\operatorname{did}(\mathrm{p}=0.005$, figure $4 \mathrm{C}$ ).

We further compared CD8 + MeTIL score with the CD8 + expression-based TIL (CD8+ ExTIL) panel, which is a molecular signature of $C D 8 B$ gene expression generated to assess CD8+ MeTIL as previously used to train other panels for evaluation of CD8+ TILs. ${ }^{19} \mathrm{We}$ determined the mRNA expression of $C D 8 B$ gene expression, encoding a part of the CD8 antigen, in SAH-SYSU cohort. Expectedly, a significant negative correlation was observed between CD8+ MeTIL score and CD8B gene expression signature $(\mathrm{r}=-0.4079, \mathrm{p}=0.008$; figure $4 \mathrm{D})$. Taken together, lower CD8+ MeTIL scores determined by QASM assay are associated with higher mean density of CD8+ T cells (CD8+ PaTILs) and increased expression of CD8B gene (CD8+ ExTIL) in CRC tissue.

Next, we evaluated the distribution of CD8+ MeTIL score determined by QASM assay by molecular features of CRC cases from the CCFR cohort. The CD8+ MeTIL score of the MSI-H group was significantly lower compared with those of the low-frequency microsatellite instability (MSIL)/microsatellite stability (MSS) group (77.8 vs 82.1, $\mathrm{p}<0.001$; figure $4 \mathrm{E}$ ), which is consistent with previous findings that MSI-H tumors harbored more CD8+ TILs than MSI-L/MSS tumors did. ${ }^{42}$ Moreover, patients with CIMP-positive (78.5 vs 81.6, $\mathrm{p}=0.048$ ) and right-side (78.9 vs $82.1, \mathrm{p}<0.001)$ tumors had significantly lower CD8+ MeTIL score compared with CIMP-negative and left-side tumors, respectively (figure $4 \mathrm{E}$ ).

\section{Prognostic value of CD8+ MeTIL in CRC and multiple cancers} Considering the role of CD8+ T-cell infiltration in tumor immune response, we sought to test the role of CD8+ MeTIL score in prognosis prediction for CRC. We used QASM assay to determine the CD8+ MeTIL scores in CRCs from the SAH-SYSU $(n=237)$ and CCFR $(n=335)$ cohorts, and grouped patients by the cut-off determined by maximally selected rank statistics (online supplemental material 1). The baseline characteristics of these grouped patients in multiple cohorts are shown in the online supplemental materials. The patients with low CD8+ MeTIL scores had significantly better OS compared with those with high CD8+ MeTIL scores in both SAH-SYSU (figure 5A; HR=0.303, 95\% CI 0.109 to $0.842, \mathrm{p}=0.022$ ) and CCFR (figure 5C; $\mathrm{HR}=3.17$, 95\% CI 1.26 to 7.99 , $\mathrm{p}=0.009$ ) cohorts. This result was further confirmed after adjusted by other significant risk factors, including age, TNM stage, KRAS mutation, and lymphovascular invasion, in multivariate Cox analysis (online supplemental material 1).

Next, we explored whether CD8+ MeTIL score could further stratify MSI-H/MSI-L/MSS patients in marker prediction assays. In survival analysis, patients were grouped according to MS status and CD8+ MeTIL score in SAH-SYSU and CCFR cohort. As expected, the death risk could be further stratified by CD8+ MeTIL score in each microsatellite subgroup (figure 5B,D). Of note, patients with MSI-H and abundant CD8+ TILs (low CD8+ MeTIL score) had the best OS in both cohorts, while MSI-L/MSS and low levels of CD8+ TILs (high CD8+ MeTIL score) predicted poor OS in both cohorts. Though this score was developed in CRC, we found the lower CD8+ MeTIL score was significantly associated with anti-PD-1 treatment response and survival benefit in non-small cell lung 
A

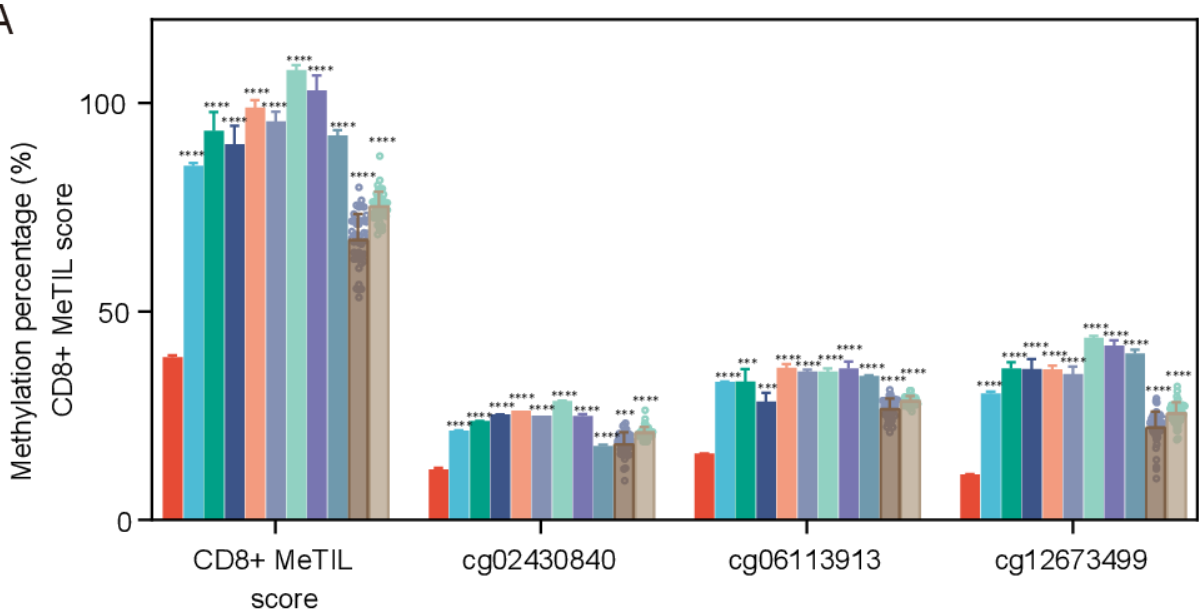

CD8+ $\mathrm{T}$ cell

CD4+ $\mathrm{T}$ cell

Monocyte

$B$ cell

RKO

SW48

HCT8

HCT15

NCM460

Colorectal cancer tissue $(n=45)$

Adjacent normal tissue $(n=44)$

B

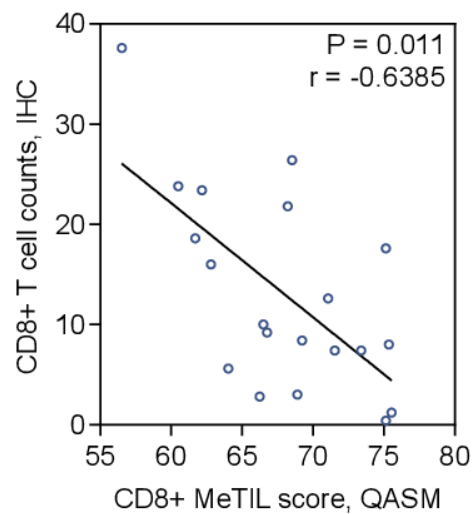

C
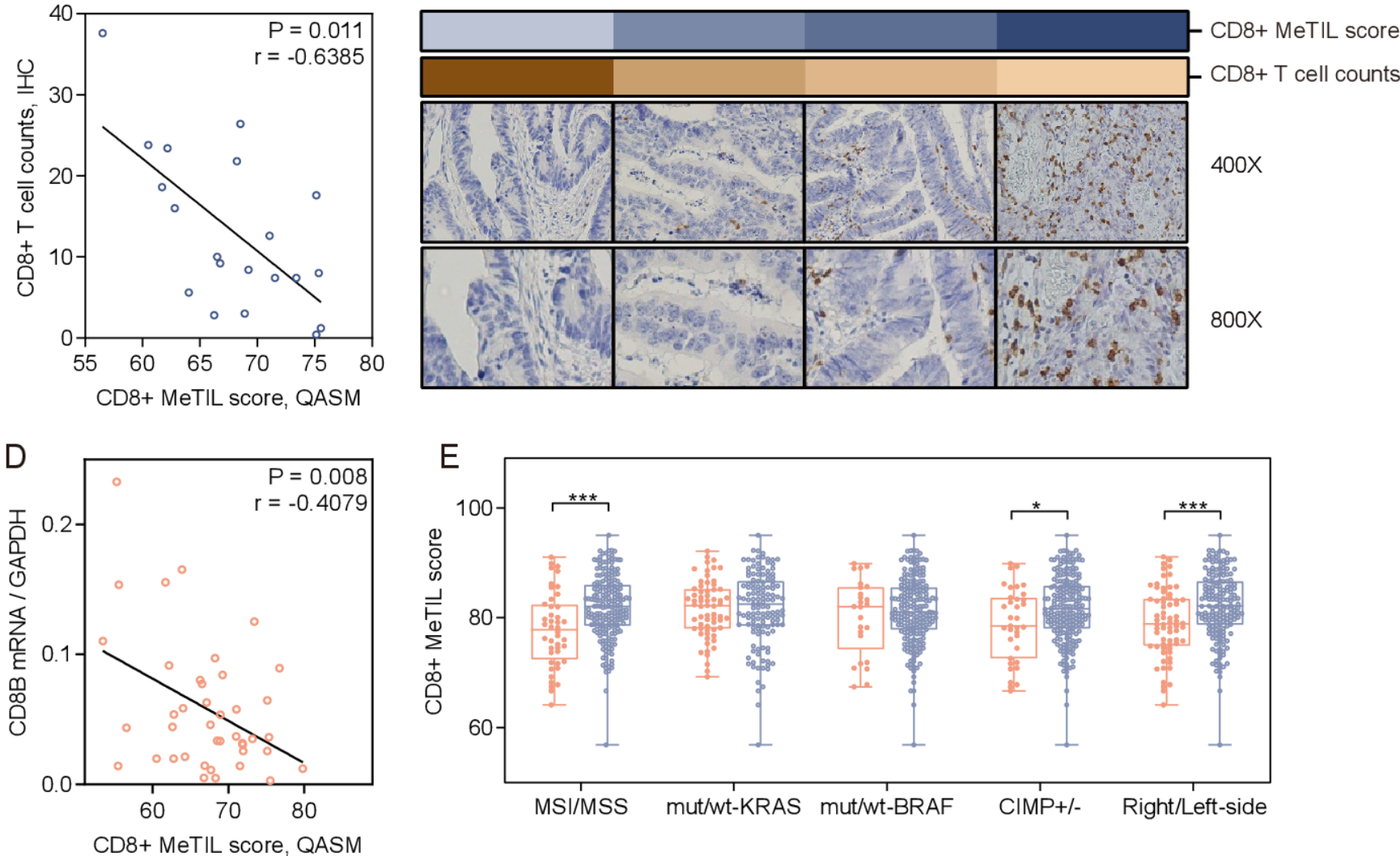

Figure 4 Evaluation of CD8 + TILs in cell lines and tumor samples using the CD8 + MeTIL score. (A) The CD8 + MeTIL score and methylation level of each CpG determined by QASM assay showed significant differences between CD8 + T cells and other immune cells, normal and cancerous colon cells. (B) Scatter plots showing the correlation between CD8 + MeTIL score determined by QASM assay and CD8 + TIL counts determined by IHC staining with FFPE slides using 23 samples from SAHSYSU cohort. Pearson's correlation coefficient and $p$ value are shown. (C) Representative images of IHC stained slides with CD8 + TIL counts from low to high (from left to right) at $\times 400$ and $\times 800$ magnification. Both the CD8 + TILs counts and CD8 + MeTIL score are shown, and a darker color means a higher value. (D) Scatter plots showing the correlation between CD8 + MeTIL determined by QASM assay and CD8 + ExTILs determined by CD8B gene mRNA expression assay using 43 samples from the SAH-SYSU cohort. Pearson's correlation coefficient and $p$ value are shown. (E) Distribution of CD8 + MeTIL by different molecular phenotypes in CCFR study cohort. The Mann-Whitney test was applied $\left({ }^{* \star} p<0.001,{ }^{*} p<0.05\right)$. Horizontal bars, median \pm IQR. CCFR, Colon Cancer Family Registry; FFPE, formalin-fixed, paraffin-embedded; SAH-SYSU, Sixth Affiliated Hospital of Sun Yat-sen University; TIL, tumor-infiltrating lymphocyte. 

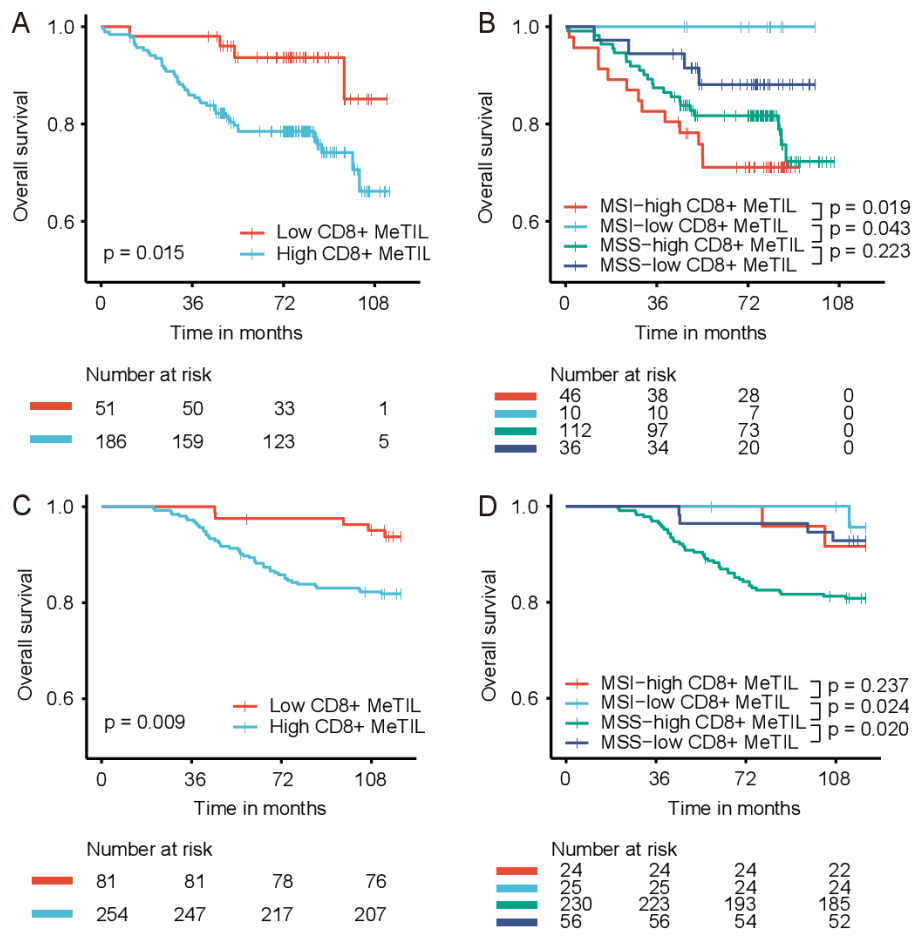

E
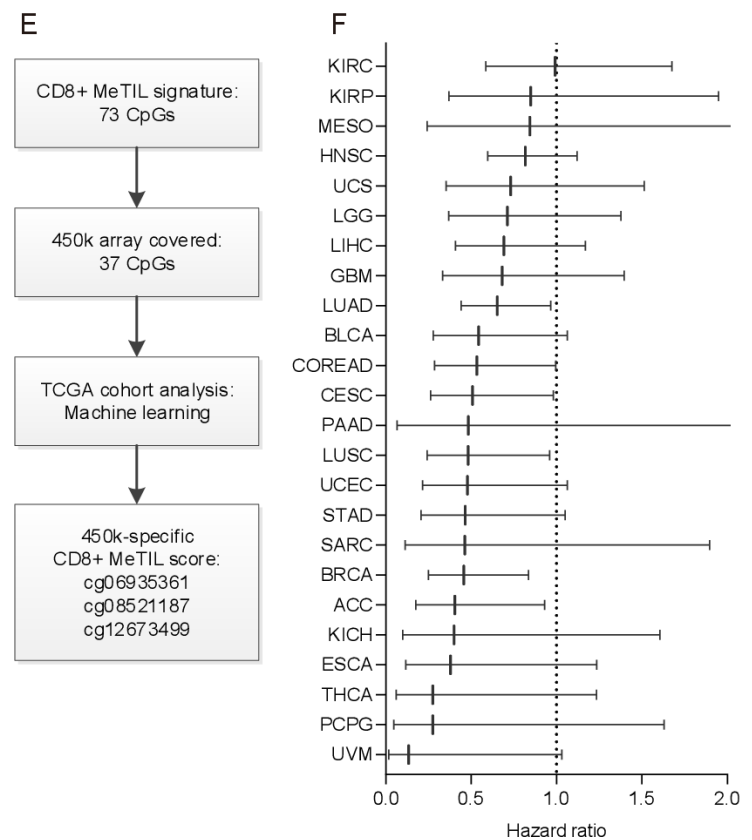
$0.991(0.586-1.675) \quad 0.973$ $0.848(0.369-1.948) \quad 0.699$ $0.844(0.242-2.942) \quad 0.790$ $0.817(0.597-1.119) \quad 0.209$ $0.731(0.353-1.512) \quad 0.399$ $0.712(0.368-1.377) \quad 0.313$ $0.690(0.408-1.169) \quad 0.169$ $0.681(0.333-1.395) \quad 0.294$ $0.652(0.441-0.965) \quad 0.033$ $0.543(0.277-1.062) \quad 0.075$ $0.533(0.285-0.994) \quad 0.048$ $0.507(0.262-0.981) \quad 0.044$ $0.482(0.066-3.517) \quad 0.472$ $0.482(0.242-0.959) \quad 0.038$ $0.478(0.215-1.062) \quad 0.070$ $0.465(0.206-1.049) \quad 0.065$ $0.462(0.113-1.896) \quad 0.284$ $0.457(0.250-0.835) \quad 0.011$ $0.404(0.176-0.929) \quad 0.033$ $0.400(0.099-1.604) \quad 0.196$ $0.379(0.116-1.234) \quad 0.107$ $0.275(0.061-1.233) \quad 0.092$ $0.275(0.046-1.628) \quad 0.155$ $0.133(0.017-1.031) \quad 0.054$

Figure 5 The prognostic value of CD8+ MeTIL in CRC and multiple cancers. Patients were grouped by CD8+ MeTIL score in SAH-SYSU cohort $(A, n=237)$ and CCFR study cohort $(C, n=335)$ based on the cut-off determined by maximally selected rank statistics. Kaplan-Meier curves of OS in each cohort with $\mathrm{p}$ values from log-rank test are shown. Patients with MSI-H and MSI-L/MSS tumors were further stratified by CD8+ MeTIL score, and four groups were generated and compared in the KaplanMeier curves of OS in the SAH-SYSU cohort (B) and the CCFR study cohort (D) with log-rank test. (E) Workflow of DMPS filtering to generate 450K-specific CD8+ MeTIL signature that could be applied in TCGA cohorts with 450K methylation array data. (F) The forest plot showed the HRs and 95\% Cls of the 450K-specific CD8+ MeTIL signature in univariate Cox analysis for survival prediction in 24 TCGA cancer types. ACC, adrenocortical carcinoma; BLCA, bladder urothelial carcinoma; BRCA, breast invasive carcinoma; CESC, cervical squamous cell carcinoma and endocervical adenocarcinoma; COREAD, colon and rectum adenocarcinoma; CRC, colorectal cancer; DMP, differentially methylated position; ESCA, esophageal carcinoma; GBM, glioblastoma multiforme; HNSC, head and neck squamous cell carcinoma; $\mathrm{KICH}$, kidney chromophobe; KIRC, kidney renal clear cell carcinoma; KIRP, kidney renal papillary cell carcinoma; LGG, brain lower-grade glioma; LIHC, liver hepatocellular carcinoma; LUAD, lung adenocarcinoma; LUSC, lung squamous cell carcinoma; meso, mesothelioma; OS, overall survival; PAAD, pancreatic adenocarcinoma; PCpG, pheochromocytoma and paraganglioma; sarc, sarcoma; STAD, stomach adenocarcinoma; SAH-SYSU, Sixth Affiliated Hospital of Sun Yat-sen University; THCA, thyroid carcinoma; UCEC, uterine corpus endometrial carcinoma; UCS, uterine carcinosarcoma; UVM, uveal melanoma. 
cancer using the EPIC methylation array data (online supplemental material 1).

Acknowledging that the CD8+ MeTIL signature was developed in CRC using EPIC methylation array data, we performed survival analysis in other cancer types using 450K methylation array data available in TCGA. In 6 of 24 tested cancer types (lung adenocarcinoma (LUAD, $\mathrm{p}=0.033$ ), lung squamous cell carcinoma (LUSC, $\mathrm{p}=0.038$ ), CRC (colon and rectum adenocarcinoma, $\mathrm{p}=0.048$ ), cervical squamous cell carcinoma (CESC, $\mathrm{p}=0.044$ ), breast invasive carcinoma (BRCA, $\mathrm{p}=0.011$ ), and adrenocortical carcinoma (ACC, $\mathrm{p}=0.033$ )), low CD8+ MeTIL scores were significantly associated with a better survival outcome (figure $5 \mathrm{~F}$ ). Taken together, these data suggest that the CD8+ MeTIL score may predict survival outcomes in multiple cancers.

\section{DISCUSSION}

In this study, we analyzed the genome-wide DNA methylation profile to identify CD8+ $\mathrm{T}$ cell-specific DMPs and constructed a CD8+ MeTIL score to assess CD8+ TILs in CRC. We demonstrated the reliability of CD8+ MeTIL signature in evaluating CD8+ TIL-based immune response by showing the tight association of this signature with IHC-based CD8+ PaTILs and CD8+ ExTIL signature. In addition, we developed a qPCR-based QASM assay that could effectively determine the CD8+ MeTIL score at single-base resolution, enabling the evaluation of CD8+ TILs in a quantitative approach. Finally, we validated the ability of CD8+ MeTIL to stratify microsatellite instability (MSI)/MSS status and prognostic outcomes in two CRC cohorts and identified associations with improved survival for several cancers from a pan-cancer analysis of the TCGA tumors.

The robustness of this study came from our presumption that cancer cells are mixed with normal epithelial cells and multiple subtypes of immune cells in each tumor block. We therefore compared the methylation profiles of CD8+ T cells with those of other potentially mixed cells and obtained 73 CD8+ T cell-specific DMPs. The genomic location of these 73 CD8+ T cell-specific DMPs showed that few CpGs were located in the high-CpG density region (CpG island), while most CpGs were located in low-CpG density regions. These regions are covered more effectively by the additional probes present in EPIC methylation array compared with the $450 \mathrm{k}$ array. Our strict selection criteria that only EPIC array datasets could be included for pooled methylation data of cell lines from published resources were an advantage of this study. Moreover, the CD8+ T cell-specific DMPs are available in DNA methylation array, which facilitates the possibility of analyzing CD8+ TILs using DNA methylation array datasets and uncovering the association with the other omics profiles.

The reliability of applying CD8+ MeTIL signature in CD8+ TILs assessment came from our cross-validation in multiple analyses. First, the GO analysis on the genes that 73 CD8+ T cell-specific DMPs were located in showed that the methylation of these CD8+ T cell-specific CpGs may participate in antitumor immunity through T-cell activation, immune effector process, immune response to tumor cell and cell killing. Therefore, we speculate that these DMPs are not merely markers for CD8+ T cell, but they may also play a role in regulating T-cell function in tumor immune response that needs to be validated in future studies. Second, we found the CD8+ MeTIL was negatively associated with T-cell exhaustion and senescence markers (online supplemental material 1), which supports the previous findings that TILs are functionally impaired with expression of exhaustion and senescence markers in tumor microenvironment. ${ }^{43-47}$ Third, the analysis of clinical and molecular features showed that low CD8+ MeTIL score was related to tumor development in the right-side colon, high differentiation, MSI-H status and wild-TP53. These clinicopathological features have been previously shown to be associated with nonmethylation-based measures of CD8+ TILs. ${ }^{4-51}$ MSI-H status had a strong association with low CD8+ MeTIL score in our CCFR cohort, which is in line with previous findings that MSI-H tumors usually harbor more CD8+ T cells due to the expression of more heteroantigens. ${ }^{51} 52$ Finally, we compared the IHC-based CD8+ PaTILs and CD8+ ExTIL results with the CD8+ MeTIL score in CRC cohorts, where both showed a significant negative correlation. This demonstrated that the signature score could be an alternative and reliable method to assess CD8+ TILs in CRC.

The current qPCR-based methylation assays have been mostly applied to determine the mean value of multiple CpGs in high-CpG density/intermediate-CpG density regions. ${ }^{37}$ Unfortunately, the DMPs included in the CD8+ MeTIL score were isolated CpGs within the low-CpG density region. Although the EPIC methylation array and bisulfite pyrosequencing can assay these isolated CpGs at single-base resolution, both of them are more expensive and time-consuming than measurement by a qPCR-based methylation assay. This may explain why the isolated CpGs within the low-CpG density region identified as methylation biomarkers in previous studies were limited to an array-analysis level and could not be extended to further study and clinical use. ${ }^{1153}$ Thus, we developed a novel qPCR-based QASM assay ${ }^{26}$ that has been shown validity in determining single-CpG methylation by an independent group. ${ }^{54}$ In the current study, we also showed its validity in measuring the methylation level of each $\mathrm{CpG}$ included in the CD8+ MeTIL score. Compared with EPIC methylation array and pyrosequencing, the QASM assay is reliable to determine the CD8+ MeTIL score. This qPCR-based method measures CD8+ TILs in an unbiased, cheap and simple manner and thus is less susceptible to variability across different cohorts. The discrepancy in CD8+ TIL evaluation between the CD8+ PaTIL and CD8+ MeTIL in the scatter plots may be partly explained by the differences in the characteristics of two methods. Considering the methylation biomarkers of cancers are widely used 
in past decades, ${ }^{55}{ }^{56}$ we anticipated that our qPCR-based assay for CD8+ MeTIL score had the potential to be widely used in clinical settings and CD8+ TIL-based research.

The current IHC assessment of CD8+ TILs is performed on tumor slides, while CD8+ MeTIL could be evaluated using both slides and tissue blocks. As a result, CD8+ MeTIL may have some potential advantages for eliminating the variations of TIL evaluation introduced from the tumor section. It is necessary to evaluate the tumor center and invasive margin, respectively, when TILs were assessed by IHC staining. ${ }^{15} 57$ However, CD8+ MeTIL is evaluated using bulk tissue from the slides or tumor blocks. The CD8+ MeTIL score of each tumor region could be precisely determined if the regions of interest could be microdissected and isolated from the slides.

It has been reported in recent studies that CD8+ TILs are associated with survival outcomes in CRC. ${ }^{12-14}$ We therefore investigated how CD8+ MeTIL score could improve risk stratification for survival outcomes in the CCFR and SAH-SYSU cohorts. As expected, the CD8+ MeTIL score showed prognostic value in these two different populations. Acknowledging that MSI-H tumors usually trigger more local immune response with the expression of more heteroantigens, ${ }^{51}{ }^{52}$ we further stratified MSI-H/MSI-L/ MSS patients by CD8+ MeTIL score. Intriguingly, patients with MSI-H and abundant CD8+ TILs (low CD8+ MeTIL score) had the best survival outcome in both cohorts, while MSI-L/MSS and deficient CD8+ TILs (high CD8+ MeTIL score) predicted poor survival outcome in both cohorts. This result suggested an anticancer role of local immune response in MSI-H tumors. Of note, the proportion of stage IV disease was low in our cohorts, and more metastatic tumors are needed to further verify the utility in metastatic disease.

It has been well documented that MSI-H status is an effective response biomarker for PD-1/PD-L1 blockade. ${ }^{4859}$ However, 20\% patients with MSI-H-CRC could not benefit from PD-1/PD-L1 blockade, and 10\% of patients with MSS-CRC also exhibited a response to PD-1/PD-L1 blockade. ${ }^{460}$ Importantly, CD8+ TILs have been reported to be a response marker for immunotherapy, ${ }^{6}$ and we did observe a subpopulation with abundant CD8+ TILs (low CD8+ MeTIL score) in the MSS/ MSI-L group. Based on the aforementioned evidence and our findings, we assume that low CD8+ MeTIL score has the potential to identify responders with MSI-L/MSS tumors, although this needs to be validated in a cohort or clinical trial with patients receiving immunotherapy.

Considering the tumor immune response is increasingly recognized to be associated with better clinical outcomes in CRC, we further examined the prognostic value of $\mathrm{CD} 8+$ MeTIL signature in multiple cancers. Interestingly, the CD8+ MeTIL signature also predicted survival outcomes in other cancers, including LUAD, LUSC, CESC, BRCA, and ACC. We speculated that this signature might predict survival outcome with improved discrimination in more tumors, including BLCA, UCEC, STAD, THCA, and UVM, if the unfiltered CD8+ MeTIL signature, as measured by the QASM assay, could have been assessed in these tumors. Of note, we demonstrated the lower CD8+ MeTIL signature could predict a response to immunotherapy in non-small cell lung cancer. These results suggested that the CD8+ MeTIL signature may also assess immune responses in other cancers and thus stratify patients for prognosis and potentially for response to immunotherapy. Considering that the CD8+ MeTIL score was generated from CRC, whether the result of this study could be extended to other tumor types might need further study to confirm.

\section{CONCLUSIONS}

In this study, we developed a DNA methylation-based CD8+ MeTIL signature that was reliable to assess CD8+ TIL-based immune response in CRC. We developed a qPCR-based QASM assay to determine the CD8+ MeTIL signature at single-base resolution and that could evaluate CD8+ TILs in a quantitative and more high-throughput way. We further validated the ability of this signature to stratify MSI/MSS status and prognostic outcomes in two CRC cohorts and TCGA cohorts with other cancers. The CD8+ MeTIL signature, if further validated, has the potential to be a useful biomarker that could be readily deployed in clinical diagnosis to inform the choice of multiple therapeutic approaches, including immunotherapy.

\section{Author affiliations}

${ }^{1}$ Guangdong Institute of Gastroenterology, Guangdong Provincial Key Laboratory of Colorectal and Pelvic Floor Disease, The Sixth Affiliated Hospital, Sun Yat-sen University, Guangzhou, China

${ }^{2}$ Department of Colorectal Surgery, The Sixth Affiliated Hospital, Sun Yat-sen University, Guangzhou, China

${ }^{3}$ Department of Colorectal and Anal Surgery, The Sixth Affiliated Hospital, Sun Yatsen University, Guangzhou, China

${ }^{4}$ Department of laboratory Medicine and Pathology, Mayo Clinic Arizona, Scottsdale, Arizona, USA

${ }^{5}$ Colorectal Oncogenomics Group, Department of Clinical Pathology, The University of Melbourne, Parkville, Victoria, Australia

${ }^{6}$ University of Melbourne Centre for Cancer Research, Victorian Comprehensive Cancer Centre, Parkville, Victoria, Australia

${ }^{7}$ Genomic Medicine and Familial Cancer Centre, The Royal Melbourne Hospital, Parkville, Victoria, Australia

${ }^{8}$ Centre for Epidemiology and Biostatistics, Melbourne School of Population and Global Health, The University of Melbourne, Parkville, Victoria, Australia

${ }^{9}$ Department of Epidemiology, University of Washington School of Public Health, Seattle, Washington, USA

${ }^{10}$ Division of Public Health Sciences, Fred Hutchinson Cancer Research Center, Seattle, Washington, USA

${ }^{11}$ Clinical Research Division, Fred Hutchinson Cancer Research Center, Seattle, Washington, USA

${ }^{12}$ Department of Medicine, University of Washington School of Medicine, Seattle, Washington, USA

Contributors Conception, design and supervision of this study; methylation array data processing and bioinformatic analysis; full access to all data for this study and take responsibility for the integrity and accuracy of the data: $\mathrm{HY}$ and $\mathrm{YL}$; experiments design and direction: $\mathrm{HY}$; data collection and interpretation: $\mathrm{HY}, \mathrm{QZ}, \mathrm{DR}$, $\mathrm{XW}, \mathrm{GT}$, and $\mathrm{YZ}$; financial support: $\mathrm{YL}, \mathrm{MH}$, and $\mathrm{HY}$; provision of study materials and patients: YL, XW, WMG, RKP, DDB, AKW, PAN, MH, BH, and DR; manuscript drafting: $\mathrm{HY}$ and QZ; manuscript revision: HY, DDB, RKP, and YL; final approval of manuscript: all authors. 
Funding This work was supported by the National Basic Research Program of China (973 Program) (number 2015CB554001); the National Natural Science Foundation of China (number 81972245, YL; number 81902877, HY); the Natural Science Fund for Distinguished Young Scholars of Guangdong Province (number 2016A030306002, YL); the Tip-top Scientific and Technical Innovative Youth Talents of Guangdong special support program (number 2015TQ01R454, YL); Sun Yat-Sen University Clinical Research 5010 Program (number 2018026, YL); the Natural Science Foundation of Guangdong Province (number 2018A0303130303, HY; number 2021A1515010134, MH); the 'Five Five' Talent Team Construction Project of the Sixth Affiliated Hospital of Sun Yat-Sen University (number P20150227202010244; number P20150227202010251, YL); the Excellent Talent Training Project of the Sixth Affiliated Hospital of Sun Yat-sen University (number R2021217202512965, YL); and the Program of Introducing Talents of Discipline to Universities, National Key Clinical Discipline (2012). The Colon CFR was supported by the National Cancer Institute and National Institutes of Health (NIH) (award U01 CA167551). Support for case ascertainment was provided in part from the Surveillance, Epidemiology, and End Results Program and the following US state cancer registries: $\mathrm{AZ}, \mathrm{CO}, \mathrm{MN}, \mathrm{NC}$, and $\mathrm{NH}$; and by the Victoria Cancer Registry (Australia) and Ontario Cancer Registry (Canada). The Colon CFR graciously thanks the generous contributions of their study subjects, dedication of study staff, and the financial support from the US National Cancer Institute, without which this important registry would not exist. The content of this manuscript does not necessarily reflect the views or policies of the NIH or any of the collaborating centers in the CCFR, nor does mention of trade names, commercial products, or organizations imply endorsement by the US Government, any cancer registry, or the CCFR.

Competing interests $\mathrm{HY}$ and coauthors are the inventors of a provisional patent filed by Sun Yat-sen University on the subject of this work (202011176816.1).

Patient consent for publication Not required.

Ethics approval This study was approved by the institutional review board of the Sixth Affiliated Hospital of Sun Yat-sen University (2017ZSLYEC-006), and written informed consent was obtained from all patients.

Provenance and peer review Not commissioned; externally peer reviewed.

Data availability statement Data are available upon reasonable request. All data relevant to the study are included in the article or uploaded as supplementary information. The data that support the findings of this study are available from the CCFR but restrictions apply to the availability of these data, which were used under license for the current study. The other datasets used and analyzed during the current study are available from the corresponding author on reasonable request.

Supplemental material This content has been supplied by the author(s). It has not been vetted by BMJ Publishing Group Limited (BMJ) and may not have been peer-reviewed. Any opinions or recommendations discussed are solely those of the author(s) and are not endorsed by BMJ. BMJ disclaims all liability and responsibility arising from any reliance placed on the content. Where the content includes any translated material, BMJ does not warrant the accuracy and reliability of the translations (including but not limited to local regulations, clinical guidelines, terminology, drug names and drug dosages), and is not responsible for any error and/or omissions arising from translation and adaptation or otherwise.

Open access This is an open access article distributed in accordance with the Creative Commons Attribution Non Commercial (CC BY-NC 4.0) license, which permits others to distribute, remix, adapt, build upon this work non-commercially, and license their derivative works on different terms, provided the original work is properly cited, appropriate credit is given, any changes made indicated, and the use is non-commercial. See http://creativecommons.org/licenses/by-nc/4.0/.

\section{ORCID iDs}

Huichuan Yu http://orcid.org/0000-0001-8357-1615

Yanxin Luo http://orcid.org/0000-0002-5200-3997

\section{REFERENCES}

1 Siegel RL, Miller KD, Jemal A. Cancer statistics, 2020. CA A Cancer $J$ Clin 2020;70:7-30.

2 Bray F, Ferlay J, Soerjomataram I, et al. Global cancer statistics 2018: GLOBOCAN estimates of incidence and mortality worldwide for 36 cancers in 185 countries. CA: A Cancer Journal for Clinicians 2018;68:394-424.

3 Ganesh K, Stadler ZK, Cercek A, et al. Immunotherapy in colorectal cancer: rationale, challenges and potential. Nat Rev Gastroenterol Hepatol 2019;16:361-75.
4 DT L, Uram JN, Wang H. Pd-1 blockade in tumors with mismatchrepair deficiency. N Engl J Med 2015;372:2509-20.

5 Overman MJ, McDermott R, Leach JL, et al. Nivolumab in patients with metastatic DNA mismatch repair-deficient or microsatellite instability-high colorectal cancer (CheckMate 142): an open-label, multicentre, phase 2 study. Lancet Oncol 2017;18:1182-91.

6 Chalabi M, Fanchi LF, Dijkstra KK, et al. Neoadjuvant immunotherapy leads to pathological responses in MMR-proficient and MMRdeficient early-stage colon cancers. Nat Med 2020;26:566-76.

7 Ray K. Colorectal cancer: evading the immune response in metastasis. Nat Rev Gastroenterol Hepatol 2018;15:191.

8 Angell HK, Bruni D, Barrett JC, et al. The immunoscore: colon cancer and beyond. Clin Cancer Res 2020;26:332-9.

9 Turksma AW, Coupé VMH, Shamier MC, et al. Extent and location of tumor-infiltrating lymphocytes in microsatellite-stable colon cancer predict outcome to adjuvant active specific immunotherapy. Clin Cancer Res 2016;22:346-56.

10 Rodriguez-Ruiz ME, Rodriguez I, Garasa S, et al. Abscopal effects of radiotherapy are enhanced by combined immunostimulatory mAbs and are dependent on CD8 T cells and Crosspriming. Cancer Res 2016;76:5994-6005.

11 Jeschke J, Bizet M, Desmedt C, et al. Dna methylation-based immune response signature improves patient diagnosis in multiple cancers. J Clin Invest 2017;127:3090-102.

12 Governa V, Trella E, Mele V, et al. The interplay between neutrophils and $\mathrm{CD} 8^{+} \mathrm{T}$ cells improves survival in human colorectal cancer. Clin Cancer Res 2017;23:3847-58.

13 Kong JC, Guerra GR, Pham T, et al. Prognostic impact of tumorinfiltrating lymphocytes in primary and metastatic colorectal cancer: a systematic review and meta-analysis. Dis Colon Rectum 2019;62:498-508.

14 Hu X, Li Y-Q, Li Q-G, et al. ITGAE defines CD8+ tumor-infiltrating lymphocytes predicting a better prognostic survival in colorectal cancer. EBioMedicine 2018;35:178-88.

15 Salgado R, Denkert C, Demaria S, et al. The evaluation of tumorinfiltrating lymphocytes (TILs) in breast cancer: recommendations by an international TILs Working group 2014. Ann Oncol 2015;26:259-71.

16 Pagès $F$, Mlecnik $B$, Marliot $F$, et al. International validation of the consensus immunoscore for the classification of colon cancer: a prognostic and accuracy study. Lancet 2018;391:2128-39.

17 Pan J-hua, Zhou H, Cooper L, et al. LAYN is a prognostic biomarker and correlated with immune infiltrates in gastric and colon cancers. Front Immunol 2019;10:6.

18 Strasser K, Birnleitner H, Beer A, et al. Immunological differences between colorectal cancer and normal mucosa uncover a prognostically relevant immune cell profile. Oncoimmunology 2019;8:e1537693.

19 Sun $\mathrm{R}$, Limkin EJ, Vakalopoulou $\mathrm{M}$, et al. A radiomics approach to assess tumour-infiltrating CD8 cells and response to anti-PD-1 or anti-PD-L1 immunotherapy: an imaging biomarker, retrospective multicohort study. Lancet Oncol 2018;19:1180-91.

20 Doi A, Park I-H, Wen B, et al. Differential methylation of tissue- and cancer-specific $\mathrm{CpG}$ island shores distinguishes human induced pluripotent stem cells, embryonic stem cells and fibroblasts. Nat Genet 2009;41:1350-3.

21 Farlik M, Halbritter F, Müller F, et al. Dna methylation dynamics of human hematopoietic stem cell differentiation. Cell Stem Cell 2016:19:808-22.

22 Stueve TR, Marconett CN, Zhou B, et al. The importance of detailed epigenomic profiling of different cell types within organs. Epigenomics 2016;8:817-29.

23 Irizarry RA, Ladd-Acosta C, Wen B, et al. The human colon cancer methylome shows similar hypo- and hypermethylation at conserved tissue-specific CpG island shores. Nat Genet 2009;41:178-86.

24 Zhou W, Dinh HQ, Ramjan Z, et al. Dna methylation loss in latereplicating domains is linked to mitotic cell division. Nat Genet 2018;50:591-602.

25 Salhab A, Nordström K, Gasparoni G, et al. A comprehensive analysis of 195 DNA methylomes reveals shared and cell-specific features of partially methylated domains. Genome Biol 2018;19:150.

26 Yu H, Bai L, Tang G, et al. Novel assay for quantitative analysis of DNA methylation at single-base resolution. Clin Chem 2019;65:664-73.

27 Newcomb PA, Baron J, Cotterchio M, et al. Colon cancer family registry: an international resource for studies of the genetic epidemiology of colon cancer. Cancer Epidemiol Biomarkers Prev 2007:16:2331-43.

28 Jenkins MA, Win AK, Templeton AS, et al. Cohort profile: the colon cancer family registry cohort (CCFRC). Int $J$ Epidemiol 2018:47:387-8. 
29 Li Y, Bai L, Yu H, et al. Epigenetic inactivation of $\alpha$-Internexin accelerates microtubule polymerization in colorectal cancer. Cancer Res 2020;80:5203-15.

30 Chen Z, Huang Z, Luo Y, et al. Genome-Wide analysis identifies critical DNA methylations within NTRKs genes in colorectal cancer. $J$ Transl Med 2021;19:73.

31 Kong X, Chen J, Xie W, et al. Defining UHRF1 domains that support maintenance of human colon cancer DNA methylation and oncogenic properties. Cancer Cell 2019;35:633-48.

32 Yeung KS, Lee TL, Mok MY, et al. Cell lineage-specific genome-wide DNA methylation analysis of patients with paediatric-onset systemic lupus erythematosus. Epigenetics 2019;14:341-51.

33 Moss J, Magenheim J, Neiman D, et al. Comprehensive human celltype methylation atlas reveals origins of circulating cell-free DNA in health and disease. Nat Commun 2018;9:5068.

34 Thompson JJ, Kaur R, Sosa CP, et al. Zbtb24 is a transcriptional regulator that coordinates with Dnmt3b to control DNA methylation. Nucleic Acids Res 2018;46:10034-51.

35 Luo Y, Wong C-J, Kaz AM, et al. Differences in DNA methylation signatures reveal multiple pathways of progression from adenoma to colorectal cancer. Gastroenterology 2014;147:418-29.

36 Price EM, Robinson WP. Adjusting for batch effects in DNA methylation microarray data, a lesson learned. Front Genet 2018;9:83.

37 Eads CA, Danenberg KD, Kawakami K, et al. MethyLight: a highthroughput assay to measure DNA methylation. Nucleic Acids Res 2000;28:E32.

38 Fortis SP, Sofopoulos M, Sotiriadou NN, et al. Differential intratumoral distributions of CD8 and CD163 immune cells as prognostic biomarkers in breast cancer. $J$ Immunother Cancer 2017;5:39.

39 Yu Z, Yu H, Zou Q, et al. Nomograms for prediction of molecular phenotypes in colorectal cancer. Onco Targets Ther 2020;13:309-21.

40 Phipps Al, Limburg PJ, Baron JA, et al. Association between molecular subtypes of colorectal cancer and patient survival. Gastroenterology 2015;148:77-87.

41 Weisenberger DJ, Levine AJ, Long TI, et al. Association of the colorectal $\mathrm{CpG}$ island methylator phenotype with molecular features, risk factors, and family history. Cancer Epidemiol Biomarkers Prev 2015;24:512-9.

42 De Rosa S, Sahnane N, Tibiletti M, et al. EBV+ and MSI gastric cancers harbor high PD-L1/PD-1 expression and high CD8+ intratumoral lymphocytes. Cancers 2018;10:102.

43 Taylor ES, McCall JL, Girardin A, et al. Functional impairment of infiltrating T cells in human colorectal cancer. Oncoimmunology 2016;5:e1234573.

44 Yang R, Cheng S, Luo N, et al. Distinct epigenetic features of tumorreactive CD8+ T cells in colorectal cancer patients revealed by genome-wide DNA methylation analysis. Genome Biol 2019;21:2.
45 Duhen T, Duhen R, Montler R, et al. Co-expression of CD39 and CD103 identifies tumor-reactive CD8 T cells in human solid tumors. Nat Commun 2018;9:2724.

46 Newman AM, Steen CB, Liu CL, et al. Determining cell type abundance and expression from bulk tissues with digital cytometry. Nat Biotechnol 2019;37:773-82.

47 Montes CL, Chapoval Al, Nelson J, et al. Tumor-induced senescent T cells with suppressor function: a potential form of tumor immune evasion. Cancer Res 2008;68:870-9.

48 Zhang D, He W, Wu C, et al. Scoring system for tumor-infiltrating lymphocytes and its prognostic value for gastric cancer. Front Immunol 2019;10.

49 Smeby J, Sveen A, Bergsland CH, et al. Exploratory analyses of consensus molecular subtype-dependent associations of TP53 mutations with immunomodulation and prognosis in colorectal cancer. ESMO Open 2019;4:e000523.

50 Guo G, Wang Y, Zhou Y, et al. Immune cell concentrations among the primary tumor microenvironment in colorectal cancer patients predicted by clinicopathologic characteristics and blood indexes. $J$ Immunother Cancer 2019;7:179.

51 Maby P, Tougeron D, Hamieh M, et al. Correlation between Density of CD8 ${ }^{+}$T-cell Infiltrate in Microsatellite Unstable Colorectal Cancers and Frameshift Mutations: A Rationale for Personalized Immunotherapy. Cancer Res 2015;75:3446-55.

52 Pakish JB, Zhang Q, Chen Z, et al. Immune microenvironment in Microsatellite-Instable endometrial cancers: hereditary or sporadic origin matters. Clin Cancer Res 2017;23:4473-81.

53 Gündert M, Edelmann D, Benner A, et al. Genome-Wide DNA methylation analysis reveals a prognostic classifier for non-metastatic colorectal cancer (ProMCol classifier). Gut 2019;68:101-10.

54 Guan R, Guo W, Hong W, et al. Identification of aberrantly methylated differentially $\mathrm{CpG}$ sites in hepatocellular carcinoma and their association with patient survival. Front Oncol 2020;10:1031.

55 Grady WM, Yu M, Markowitz SD. Epigenetic alterations in the gastrointestinal tract: current and emerging use for biomarkers of cancer. Gastroenterology 2021;160:690-709.

56 Jung G, Hernández-Illán E, Moreira L, et al. Epigenetics of colorectal cancer: biomarker and therapeutic potential. Nat Rev Gastroenterol Hepatol 2020;17:111-30.

57 Galon J, Costes A, Sanchez-Cabo F, et al. Type, density, and location of immune cells within human colorectal tumors predict clinical outcome. Science 2006;313:1960-4.

58 Havel JJ, Chowell D, Chan TA. The evolving landscape of biomarkers for checkpoint inhibitor immunotherapy. Nat Rev Cancer 2019:19:133-50.

59 Duffy MJ, Crown J. Biomarkers for predicting response to immunotherapy with immune checkpoint inhibitors in cancer patients. Clin Chem 2019;65:1228-38.

60 Dudley JC, Lin M-T, Le DT, et al. Microsatellite instability as a biomarker for PD-1 blockade. Clin Cancer Res 2016;22:813-20. 